\title{
A TEST OF INDEPENDENCE BASED ON THE $(r, s)$-DIRECTED DIVERGENCE
}

\author{
D. MORALES, L. PARDO, M. SALICRU AND M. L. MENÉNDEZ
}

\begin{abstract}
Directed divergence statistics quantifies the divergence between a joint probability measure and the product of its marginal probabilities on the basis of contingency tables. Asymptotic properties of these statistics are investigated either considering random sampling or stratified random sampling with proportional allocation and independence among strata. To finish some tests of hypotheses of independence are presented.
\end{abstract}

\section{Introduction}

Let $(X, Y)$ be a random variable of the discrete type taking on pairs of values $\left(x_{i}, y_{j}\right), i=1, \ldots, M$, and $j=1,2, \ldots, \mathbb{K}$. We denote

$$
P_{X Y}=\left(p_{i j}\right)_{\substack{i=1, \ldots, M \\ j=1, \ldots, K}}=\left(P\left(X=x_{i}, Y=y_{j}\right)\right)_{\substack{i=1, \ldots, M \\ j=1, \ldots, K}}
$$

the joint probability mass function of $(X, Y)$ and by $P_{X}=\left(p_{i:}\right)_{i=1, \ldots, M}$ and $P_{Y}=$ $\left(p_{. j}\right)_{j=1, \ldots, K}$ the corresponding marginal probability distributions, respectively, i.e. $p_{i .}=\sum_{j=1}^{K} p_{i j}$ and $p_{. j}=\sum_{i=1}^{M} p_{i j}$.

Kullback and Leibler (1951) first introduced a measure of information con-

This work was partially supported by the direccion general de investigacion cientifica y/ tecnica (Dgicyt) under the contract ps89-0019.

Received September 30, 1991. 
cerning to $P_{X Y}$ and $P_{X} * P_{Y}=\left(p_{i . p}, j\right)_{\substack{i=1, \ldots, M, j=1, \ldots, K}}$, as

$$
D\left(\mathbb{P}_{X Y} \| \mathbb{P}_{X} * \mathbb{P}_{Y}\right)=\sum_{i=1}^{M} \sum_{j=1}^{K} p_{i j} \log \frac{p_{i j}}{p_{i . P_{. j}}}
$$

Renyi (1961) first presented a generalization of (1), as

$$
D_{r}^{1}\left(P_{X Y} \| P_{X} * P_{Y}\right)=(r-1)^{-1} \log \left[\sum_{i=1}^{M} \sum_{j=1}^{K} p_{i j}^{r}\left(p_{i .} . j\right)^{1-r}\right], r \neq 1, r>0
$$

Another well known generalization of (1) is given by

$$
D_{s}^{s}\left(P_{X Y} \| P_{X} * P_{Y}\right)=(s-1)^{-1}\left[\sum_{i=1}^{M} \sum_{j=1}^{K} p_{i j}^{s}\left(p_{i .} p_{. j}\right)^{1-s}-1\right]
$$

The following limits are easy to check

$$
\lim _{r \rightarrow 1} D_{r}^{1}\left(P_{X Y} \| P_{X} * P_{Y}\right)=\lim _{s \rightarrow 1} D_{s}^{s}\left(P_{X Y} \| P_{X} * \mathbb{P}_{Y}\right)=D\left(\mathbb{P}_{X Y} \| \mathbb{P}_{X} * P_{Y}\right)
$$

Sharma and Mittal (1975) studied the following two generalizations

$$
\begin{array}{r}
D_{1}^{s}\left(P_{X Y} \| P_{X} * P_{Y}\right)=(s-1)^{-1}\left[\exp \left[(s-1) D\left(P_{X Y} \| P_{X} * P_{Y}\right)\right]-1\right] s \neq 1 \\
D_{r}^{s}\left(P_{X Y} \| P_{X} * P_{Y}\right)=(s-1)^{-1}\left[\left[\sum_{i=1}^{M} \sum_{j=1}^{K} p_{i j}^{r}\left(p_{i . p . j}\right)^{1-r}\right] \frac{s-1}{r-1}-1\right] \\
r \neq 1, s \neq 1, r>0(5)
\end{array}
$$

Again, we can easily verify the following limits:

$$
\begin{aligned}
\lim _{s \rightarrow 1} D_{r}^{s}\left(P_{X Y} \| P_{X} * P_{Y}\right) & =D_{r}^{1}\left(P_{X Y} \| P_{X} * P_{Y}\right) \\
\lim _{s \rightarrow 1} D_{1}^{s}\left(P_{X Y} \| P_{x} * P_{Y}\right) & =D\left(P_{X Y} \| P_{X} * P_{Y}\right)
\end{aligned}
$$

when $r=s$ in (5) we have

$$
D_{r}^{s}\left(P_{X Y} \| P_{X} * P_{Y}\right)=D_{s}^{s}\left(P_{X Y} \| P_{X} * \mathbb{P}_{Y}\right)
$$


In this paper we analyze the properties of the analogue estimate of $D_{r}^{s}\left(P_{X Y} \|\right.$ $\left.P_{X} * P_{Y}\right)$ in a random sampling as well as its application to testing statistical hypotheses. Taking limits the results obtained are also valid for the divergences $D_{1}^{s}\left(P_{X Y} \| P_{X} * P_{Y}\right), D_{r}^{1}\left(P_{X Y} \| P_{X} * P_{Y}\right)$ and $D\left(P_{X Y} \| P_{X} * P_{Y}\right)$.

\section{Asymptotic distribution of $D_{r}^{s}\left(\hat{P}_{X Y} \| \hat{P}_{X} * \hat{P}_{Y}\right)$}

Consider a sample of $n$ members drawn at random with replacement from the population. We denote by $\hat{p}_{i j}=n_{i j} / n, \hat{p}_{i .}=n_{i .} / n, \hat{p}_{. j}=n_{. j} / n$ the sample estimators of $p_{i j}, p_{i}$. and $p_{j}$, where $n_{i j}$ is the number of observations of the value $\left(x_{i}, y_{j}\right)(i=1, \ldots, M, j=1 \ldots, K)$ in the sample, $n_{i .}=\sum_{j=1}^{K} n_{i j}$ and $n_{. j}=\sum_{i=1}^{M} n_{i j}$. The $(r, s)$-directed divergence in the sample may be quatified as follows:

$$
\begin{aligned}
& D_{r}^{s}\left(\hat{P}_{X Y} \| \hat{P}_{X} * \hat{P}_{Y}\right)=(s-1)^{-1}\left[\left[\sum_{i=1}^{M} \sum_{j=1}^{K} \hat{p}_{i j}^{r}\left(\hat{p}_{i .} \hat{p}_{. j}\right)^{1-r}\right]^{\frac{s-1}{r-1}}-1\right] \\
& r \neq 1, s \neq 1, r>0 .
\end{aligned}
$$

the other divergence measures in the sample are given by

$$
\begin{aligned}
& \lim _{s \rightarrow 1} D_{r}^{s}\left(\hat{P}_{X Y} \| \hat{P}_{X} * \hat{P}_{Y}\right)=D_{r}^{1}\left(\hat{P}_{X Y} \| \hat{P}_{X} * \hat{P}_{Y}\right) \\
& \lim _{r \rightarrow 1} D_{r}^{s}\left(\hat{P}_{X Y} \| \hat{P}_{X} * \hat{P}_{Y}\right)=D_{1}^{s}\left(\hat{P}_{X Y} \| \hat{P}_{X} * \hat{P}_{Y}\right)
\end{aligned}
$$

$\lim _{r \rightarrow 1} D_{r}^{1}\left(\hat{P}_{X Y} \| \hat{P}_{X} * \hat{P}_{Y}\right)=\lim _{s \rightarrow 1} D_{1}^{s}\left(\hat{P}_{X Y} \| \hat{P}_{X} * \hat{P}_{Y}\right)=D\left(\hat{P}_{X Y} \| \hat{P}_{X} * \hat{P}_{Y}\right)$

When the sample is drawn at random and with replacement from the population, the random vector $\left(n \hat{p}_{11}, \ldots, \hat{p}_{M K}\right)$ has a multinomial distribution with parameters $\left(n ; p_{11}, \ldots, p_{M K}\right)$.

The asymptotic distribution of $D_{r}^{s}\left(\hat{P}_{X X} \| \hat{P}_{X} * \hat{P}_{Y}\right)$ in the random sampling is given in the following theorem:

Theorem 1. If we consider the analogue estimate $D_{r}^{s}\left(\hat{P}_{X / Y} \| \hat{P}_{X} * \hat{P}_{Y}\right)$ obtained by replacing $p_{i j}, p_{i .}$ and $p_{. j}$ by the observed frequencies $\hat{p}_{i j}, \hat{p}_{i .}$ and $\hat{p}_{. j}$, 
then

$$
n^{\frac{1}{2}}\left[D_{r}^{s}\left(\hat{P}_{X Y} \| \hat{P}_{X} * \hat{P}_{Y}\right)-D_{r}^{s}\left(\mathbb{P}_{X Y} \| \mathbb{P}_{X} * \mathbb{P}_{Y}\right)\right] \underset{n_{\uparrow} \infty}{\stackrel{L}{L}} \mathbb{N}\left(0, v_{r, s}^{2}\right)
$$

where

$$
v_{r, s}^{2}=\sum_{i=1}^{M} \sum_{j=1}^{K} p_{i j} \frac{\partial}{\partial p_{i j}} h_{r}^{s}\left(p_{11}, \ldots, p_{M K}\right)^{2}-\left[\sum_{i=1}^{M} \sum_{j=1}^{K} p_{i j} \frac{\partial}{\partial p_{i j}} h_{r}^{s}\left(p_{11}, \ldots, p_{M K}\right)\right]^{2}
$$

and

$$
\frac{\partial}{\partial p_{i j}} h_{r}^{s}\left(p_{11}, \ldots, p_{M K}\right)=\left\{\begin{array}{c}
\frac{1}{r-1}\left[\sum_{i=1}^{M} \sum_{j=1}^{K} p_{i j}^{r}\left(p_{i . p} p_{. j}\right)^{1-r}\right]^{\frac{s-r}{r-1}}\left\{r p_{i j}^{r-1}\left(p_{i . j} p_{. j}\right)^{1-r}\right. \\
+(1-r) \sum_{j=1}^{K} p_{i j}^{r} p_{i .}^{-r} p_{. j}^{1-r}+(1-r) \sum_{i=1}^{M} p_{i j}^{r} p_{. j}^{-r} \\
\left.p_{i . r}^{1-r}\right\} \\
\exp \left[(s-1) \sum_{i=1}^{M} \sum_{j=1}^{K} p_{i j} \log \frac{p_{i j}}{p_{i . p . j}}\right]\left[\log \frac{p_{i j}}{\left.p_{i . p . j}-1\right]}\right. \\
r=1
\end{array}\right.
$$

Proof. Bickel and Doksum $(1977, \mathrm{pp} .135)$ have shown that if $\frac{\partial}{\partial x_{i}} h\left(x_{1}, \ldots\right.$, $x_{l}$ ) exist and is continuous for all $i=1, \ldots, l$, then the asymptotic distribution of $T_{n}=h\left(\hat{p}_{1}, \ldots, \hat{p}_{l}\right)$ in a random sampling is given by

$$
n^{\frac{1}{2}}\left[T_{n}-h\left(p_{1}, \ldots, p_{l}\right)\right] \underset{n_{\uparrow} \infty}{\stackrel{L}{\longrightarrow}} \mathcal{N}\left(0, \sigma^{2}\right)
$$

where

$$
\sigma^{2}=\sum_{i=1}^{l} p_{i}\left[\frac{\partial h}{\partial p_{i}}\left(p_{1}, \ldots, p_{l}\right)\right]^{2}-\left[\sum_{i=1}^{l} p_{i} \frac{\partial h}{\partial p_{i}}\left(p_{1}, \ldots, p_{l}\right)\right]^{2}
$$

The derivatives are calculated treating $p_{1}, \ldots, p_{l}$ as "independent variables" not linked by $p_{1}+p_{2}+\ldots+p_{l}=1$. If we consider the function

$$
h\left(p_{11}, \ldots, p_{M K}\right)=D_{s}^{r}\left(\mathbb{P}_{X Y} \| P_{X} * \mathbb{P}_{Y}\right)
$$

we obtain the enunciated result, $r \neq 1$ and $s \neq 1$. By continuity of the variance we obtain the ennunciated result for $r>0$ and $s \in(-\infty, \infty)$. 
Theorem 2. If $\mathbb{P}_{X Y}=\mathbb{P}_{X} * \mathbb{P}_{Y}$, then

$$
\frac{2 n D_{r}^{s}\left(\hat{P}_{X Y} \| \hat{P}_{X} * \hat{P}_{Y}\right)}{r} \underset{n_{\uparrow} \infty}{\stackrel{L}{\longrightarrow}} \chi_{(M-1)(K-1)}^{2}
$$

Proof. Consider the function $\phi(x)=x^{r}$. A Taylor's expansion of $\phi(x)$ around the point 1 for $x=\left(\hat{p}_{i j}\right) /\left(\hat{p}_{i .} \hat{p}_{. j}\right)$ yields

$$
\phi\left[\frac{\hat{p}_{i j}}{\hat{p}_{i . \hat{p}_{. j}}}\right]=1+\left[\frac{\hat{p}_{i j}}{\hat{p}_{i . \hat{p}_{. j}}}-1\right] r+\frac{1}{2}\left[\frac{\hat{p}_{i j}}{\hat{p}_{i . \hat{p}_{. j}}}-1\right]^{2}\left[r(r-1)+\varepsilon_{i j n}\right]
$$

$i=1, \ldots, M, j=1, \ldots, \mathbb{K}$, where $\varepsilon_{i, j, n} \frac{P}{n_{\uparrow} \infty} 0$ as $n \rightarrow \infty$.

Multiplying both sides of the last expression by $\hat{p}_{i .} \hat{p}_{. j}$ and suming over $i=$ $1, \ldots, M, j=1, \ldots, \mathbb{K}$, we get

$$
\begin{aligned}
\sum_{i=1}^{M} \sum_{j=1}^{K} \hat{p}_{i j}^{r}\left(\hat{p}_{i .} \hat{p}_{. j}\right)^{1-r}= & +\frac{1}{2} r(r-1) \sum_{i=1}^{M} \sum_{j=1}^{K}\left[\frac{\hat{p}_{i j}}{\hat{p}_{i . \hat{p}_{. j}}}-1\right]^{2} \hat{p}_{i . \hat{p}_{. j}} \\
& +\frac{1}{2} \sum_{i=1}^{M} \sum_{j=1}^{K}\left[\frac{\hat{p}_{i j}}{\hat{p}_{i . \hat{p}_{. j}}}-1\right]^{2} \hat{p}_{i . \hat{p}_{. j} \varepsilon_{i j n}=1+x_{n}}
\end{aligned}
$$

Using the binomial expansion of $\left[1+x_{n}\right]^{\frac{s-1}{r-1}}$, substracting 1 and multiplying by $\frac{2 n}{r(s-1)}$, we obtain $\frac{2 n D_{r}^{s}\left(\hat{P}_{X Y} \| \hat{P}_{X} * \hat{P}_{Y}\right)}{r}=n \sum_{i=1}^{M} \sum_{j=1}^{K} \frac{1}{\hat{p}_{i . \hat{p}_{. j}}}\left[\hat{p}_{i j}-\hat{p}_{i .} \hat{p}_{. j}\right]^{2}+$ $\frac{n}{r(r-1)} \sum_{i=1}^{M} \sum_{j=1}^{K} \frac{\left(\hat{p}_{i j}-\hat{p}_{\left.i . \hat{p}_{. j}\right)^{2}}\right.}{p_{i . p . j}} \varepsilon_{i j n}+\frac{2(s-1)}{r(r-1)} n x_{n} \varepsilon_{n}^{\prime}$, where $\varepsilon_{n}^{\prime} \frac{P}{n \uparrow \infty} 0$.

Note that

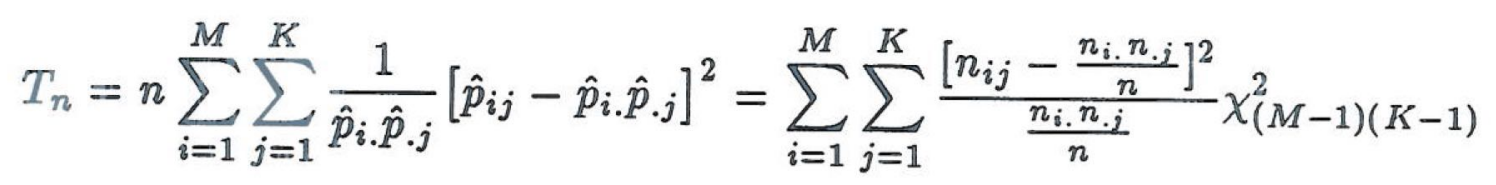

which is the well known limit distribution of the classical statistic to test independence in contingency tables.

As $n x_{n}=\frac{1}{2} r(r-1) T_{n}+\frac{1}{2} T_{n} \varepsilon_{i j n}$, then $n x_{n}$ converges in law to $\frac{1}{2} r(r-$ 1) $\chi_{(M-1)(K-1)}^{2}$ and $n x_{n} \varepsilon_{n}^{\prime} \underset{n_{\uparrow} \infty}{\stackrel{P}{\longrightarrow}} 0$. So the result follows. 
Now we suppose that the population associated with the bidimensional random variable $(X, Y)$ is finite, $N$ elements, and it can divided into $L$ nonoverlapping subpopulations, called strata as homogeneous as possible. Let $N_{j}$ be the number of individuals into the $j$ th stratum (so that $\sum_{j=1}^{L} N_{j}=N$ ) and let $p_{i j l}$ be the probability that a randomly selected member belongs to the $l$ th stratum and takes on the value $\left(x_{i}, y_{j}\right), i=1, \ldots, M, j=1, \ldots, k, l=1, \ldots, L$. Thus

$$
\sum_{i=1}^{M} \sum_{j=1}^{K} p_{i j l}=\frac{N_{l}}{N}, \quad \sum_{i=1}^{M} \sum_{j=1}^{K} \sum_{l=1}^{L} p_{i j k}=1
$$

Let $p_{i j}$. be the probability that a randomly selected number in the whole population takes on the value $\left(x_{i}, y_{j}\right), p_{i j}=\sum_{l=1}^{L} p_{i j l} i=1, \ldots, M$ and $j=1, \ldots, \mathbb{K}$, $p_{i . l}=\sum_{j=1}^{K} p_{i j l}$ the marginal probability of the value $x_{i}$ in the lth stratum and $p_{. j l}=\sum_{i=1}^{M} p_{i j l}$ the marginal probability of the value $y_{j}$ in the $l$ th stratum. The $(r, s)$-directed divergence in this context is given by

$$
\begin{aligned}
&{ }^{s t} D_{r}^{s}\left(P_{X Y} \| P_{X} * P_{Y}\right) \\
&=(s-1)^{-1}\left\{\left[\sum_{i=1}^{M} \sum_{j=1}^{K}\left[\sum_{l=1}^{L} p_{i j l}\right]^{r}\left[\sum_{l=1}^{L} p_{i . l}\right]^{1-r}\left[\sum_{l=1}^{L} p_{. j l}\right]^{1-r}\right]^{\frac{s-1}{r-1}}-1\right\} \\
& r \neq 1, s \neq 1, r>0
\end{aligned}
$$

The other divergence measures in the stratified sampling are giving by

$$
\begin{aligned}
& \lim _{s \rightarrow 1}{ }^{s t} D_{r}^{s}\left(P_{X Y} \| P_{X} * P_{Y}\right)={ }^{s t} D_{r}^{1}\left(P_{X Y} \| P_{X} * P_{Y}\right) \\
& \lim _{r \rightarrow 1}{ }^{s t} D_{r}^{s}\left(P_{X Y} \| P_{X} * P_{Y}\right)={ }^{s t} D_{1}^{s}\left(P_{X Y} \| P_{X} * P_{Y}\right)
\end{aligned}
$$

$\lim _{r \rightarrow 1}{ }^{s t} D_{r}^{1}\left(P_{X Y} \| P_{X} * P_{Y}\right)=\lim _{s \rightarrow 1}{ }^{s t} D_{1}^{s}\left(P_{X Y} \| P_{X} * P_{Y}\right)={ }^{s t} D\left(P_{X * Y} \| P_{X} * P_{Y}\right)$

Now we suppose that a stratified sample of size $n$ is drawn at random from the population independently in different strata. We hereafter suppose that the sample is choosen by proportional allocation in each stratum. Assume also that a sample of size $n_{k}$ is drawn independently at random with replacement from the $l$ th stratum where $n_{l} / n=N_{l} / N$. If $\hat{p}_{i j k}$ denotes the relative frequency of the values $\left(x_{i}, y_{j}\right)$ into the $l$ th stratum (and hence $\sum_{i=1}^{M} \sum_{j=1}^{K} p_{i j k}=n_{l} / n$ ), 
$\hat{p}_{i . l}=\sum_{j=1}^{K} \hat{p}_{i j l}$ and $\hat{p}_{. j l}=\sum_{i=1}^{M} \hat{p}_{i j k}$, the $(r, s)$-directed divergence in the sample may be quantified by ${ }^{s t} \mathbb{D}_{r}^{s}\left(\hat{P}_{X Y} \| \hat{P}_{X} * P_{Y}\right)$. In this context we establish the following theorem.

\section{Theorem 3.}

$$
n^{\frac{1}{2}}\left[{ }^{s t} D_{r}^{s}\left(\hat{P}_{X Y} \| \hat{P}_{X} * \hat{P}_{Y}\right)-{ }^{s t} D_{r}^{s}\left(P_{X Y} \| P_{X} * P_{Y}\right)\right] \underset{n_{\uparrow} \infty}{\stackrel{L}{L}} \mathcal{N}\left(0,{ }^{s t} v^{2}\right)
$$

where

$$
{ }^{s t} v^{2}=\sum_{i=1}^{M} \sum_{j=1}^{K}{ }_{s}^{r} d_{i j} p_{i j .}-\sum_{l=1}^{L} \frac{N}{N_{l}}\left[\sum_{i=1}^{M} \sum_{j=1}^{K}{ }_{s}^{r} d_{i j} p_{i j l}\right]^{2}
$$

and

$$
{ }_{s}^{r} d_{i j}=\left\{\begin{array}{l}
\frac{1}{r-1}\left[\sum_{i=1}^{M} \sum_{j=1}^{K} p_{i j .}^{r}\left(p_{i . .} p_{. j .}\right)^{1-r}\right]^{\frac{s-r}{r-1}}\left\{r p_{i j .}^{r-1}\left(p_{i . . . p . j .}\right)^{1-r}\right. \\
\left.\quad+(1-r) \sum_{j=1}^{K} p_{i j .}^{r} p_{i . .}^{-r} p_{. j .}^{1-r}+(1-r) \sum_{i=1}^{M} p_{i j .}^{r} p_{. j .}^{-r} p_{i . .}^{1-r}\right\}, r \neq 1 \\
\exp \left\{(s-1) \sum_{i=1}^{M} \sum_{j=1}^{K} p_{i j .} \log \frac{p_{i j .}}{p_{i . . p . j .}}\right\}\left[\log \frac{p_{i j .}}{p_{i . . p . j .}}-1\right], r=1
\end{array}\right.
$$

Proof. First, we prove the result for $r \neq 1, s \neq 1$. Consider the vector $a=\left(a_{i j k}, i=1, \ldots, M ; j=1, \ldots, K ; l=1, \ldots, L\right)$ with $a_{M K L}(l=1, \ldots, L)$ excluded and the function

$$
\begin{aligned}
g_{r}^{s}(a)= & (s-1)^{-1}\left\{\left[\sum_{l=1}^{L}\left[a_{i j l}\right]^{r}\left[\left[\sum_{h=1}^{K} \sum_{l=1}^{L} a_{i h l}\right]\left[\sum_{h=1}^{M} \sum_{l=1}^{L} a_{h j l}\right]\right]^{1-r}\right.\right. \\
& (i, j) \in A=\{(i, j) /(i, j) \neq(M, K), i=1, \ldots, M, j=1, \ldots, K\} \\
& +\left[1-\sum_{(i, j) \in A}\left[\sum_{l=1}^{L} a_{i j l}\right]\right]^{r}\left[1-\sum_{j=1}^{K-1}\left[\sum_{l=1}^{L} \sum_{i=1}^{M} a_{i h l}\right]\right]^{1-r} \\
& \left.\left.\cdot\left[1-\sum_{i=1}^{M-1}\left[\sum_{l=1}^{L} \sum_{j=1}^{K} a_{i j l}\right]\right]^{1-r}\right]^{\frac{s-1}{r-1}}-1\right\} r \neq 1, s \neq 1
\end{aligned}
$$

Let

$\hat{P}_{*}=\left(\hat{p}_{i j l}, i=1, \ldots, M ; j=1, \ldots, K ; l=1, \ldots, L\right)$ with $\hat{p}_{M K l}(l=1, \ldots, L)$ excluded, and 
$P_{*}=\left(p_{i j k}, i=1, \ldots, M ; j=1, \ldots, K ; l=1, \ldots, L\right)$ with $p_{M K l}(l=1, \ldots, L)$ excluded.

Let us also define

$$
\hat{P}=\left(\hat{p}_{i j l}, i=1, \ldots, M ; j=1, \ldots, \mathbb{K} ; l=1, \ldots, L\right)
$$

and

$$
P=\left(p_{i j l}, i=1, \ldots, M ; j=1, \ldots, K ; l=1, \ldots, L\right) .
$$

If we consider the Taylor's expansion of $g_{r}^{s}\left(\hat{\mathbb{P}}_{*}\right)$ in a neigbourhood of $\boldsymbol{P}_{*}$, we obtain that

$$
n^{\frac{1}{2}}\left[g_{r}^{s}(\hat{P})-g_{r}^{s}(P)\right]=n^{\frac{1}{2}}\left[{ }^{s t} D_{r}^{s}\left(\hat{P}_{X Y} \| \hat{P}_{X} * \hat{P}_{Y}\right)-{ }^{s t} D_{r}^{s}\left(P_{X Y} \| P_{X} * P_{Y}\right)\right]
$$

and

$$
n^{\frac{1}{2}} \sum_{i=1}^{M} \sum_{j=1}^{K} \sum_{l=1}^{L} \frac{\partial g_{r}^{s}(P)}{\partial p_{i j l}}\left[\hat{p}_{i j l}-p_{i j l}\right]
$$

have asymptotically the same p.d.f..

The random vectors

$$
\left[n \hat{p}_{11 l}, \ldots, n \hat{p}_{M k l}\right], \quad l=1, \ldots, L
$$

are independent and multiomial distributed with parameters

$$
\left[n_{l} ; \frac{N}{N_{l}} p_{11 l}, \ldots, \frac{N}{N_{l}} p_{M k l}\right], \quad l=1, \ldots, L
$$

Applying the $M K$-dimensional Central Limit Theorem, we obtain

$$
\begin{aligned}
n_{l}^{\frac{1}{2}}\left[\left[\frac{n}{n_{l}} \hat{p}_{11 l}-\frac{N}{N_{l}} p_{11 l}\right], \ldots,\left[\frac{n}{n_{l}} \hat{p}_{M K l}-\frac{N}{N_{l}} p_{M K l}\right]\right] \underset{n_{\uparrow} \infty}{\stackrel{L}{\longrightarrow} \mathcal{N}(0, \Sigma(l))} \\
\quad l=1, \ldots, L
\end{aligned}
$$

where

and

$$
\sum(l)=\left[\frac{N}{N_{l}} p_{\left(i_{1}, j_{1}\right) l}\left(\delta_{\left(i_{1}, j_{1}\right)\left(i_{2}, j_{2}\right)}-\frac{N}{N_{l}} p_{\left(i_{2}, j_{2}\right) l}\right)\right]_{\substack{i_{1}, i_{2}=1, \ldots, M \\ j_{1}, j_{2}=1, \ldots, K}}
$$




$$
\delta_{\left(i_{1}, j_{1}\right)\left(i_{2}, j_{2}\right)}=1 \text { if }\left(i_{1}=i_{2}, j_{1}=j_{2}\right) \text { and } \delta_{\left(i_{1}, j_{1}\right)\left(i_{2}, j_{2}\right)}=0 \text { otherwise }
$$

As $n / n_{l}=N / N_{l}$ and $n_{l}^{1 / 2}=n^{1 / 2}\left(N_{l} / N\right)^{1 / 2}$, we have

$$
\begin{aligned}
X_{l}^{t}=n^{\frac{1}{2}}\left[\frac{N}{N_{l}}\right]^{\frac{1}{2}}\left[\left(\hat{p}_{11 l}-p_{11 l}\right), \ldots,\left(\hat{p}_{M K l}-p_{M K l}\right)\right] \underset{n_{\uparrow} \infty}{\stackrel{L}{\longrightarrow}} \mathbb{N}(0, \Sigma(l)) . & \\
l & =1, \ldots, L
\end{aligned}
$$

Therefore, the asymptotic probability distribution function of the linear function $b_{l}^{t} X_{l}$, where

$$
b_{l}^{t}=\left[\frac{\partial g_{r}^{s}(\mathbb{P})}{\partial p_{11 l}}, \ldots, \frac{\partial g_{r}^{s}(\mathbb{P})}{\partial p_{M K l}}\right], \quad l=1, \ldots, \mathbb{L}
$$

is normal with mean zero and variance $b_{l}^{t} \Sigma(l) b_{l}$.

As $X_{1}, X_{2}, \ldots, X_{L}$ are independent vectors,

$$
\sum_{l=1}^{L}\left[\frac{N_{l}}{N}\right]^{\frac{1}{2}} b_{l}^{t} X_{l}^{t}=n^{\frac{1}{2}} \sum_{l=1}^{L} \sum_{i=1}^{M} \sum_{j=1}^{K} \frac{\partial g_{r}^{s}(\mathbb{P})}{\partial p_{i j l}}\left(\hat{p}_{i j l}-p_{i j l}\right)
$$

has a normal asymptotic probability distribution function with mean zero and variance

$$
{ }^{s t} v^{2}=\frac{1}{N} \sum_{l=1}^{L} N_{l} b_{l}^{t} \Sigma(l) b_{l}
$$

Now we calculate ${ }^{s t} v^{2}$ explicitly. As

$$
\begin{aligned}
\frac{\partial g_{r}^{s}(P)}{\partial p_{i j l}}= & \frac{1}{r-1}\left[\sum_{i=1}^{M} \sum_{j=1}^{K} p_{i j .}^{r}\left(p_{i . . p . j .}\right)^{1-r}\right]^{\frac{s-r}{r-1}}\left\{r p_{i j .}^{r-1}\left(p_{i . .} p_{. j .}\right)^{1-r}\right. \\
& \left.+(1-r) \sum_{j=1}^{K} p_{i j .}^{r} ._{i . .}^{-r} p_{. j .}^{1-r}+(1-r) \sum_{i=1}^{M} p_{i j . ._{. j .}^{r} p^{-r} p_{i . .}^{1-r}}^{1-r}\right\}
\end{aligned}
$$

we obtain the expression of ${ }^{s t} v^{2}$ for $r \neq 1$.

Now, by contynuity of ${ }^{s t} v$ we obtain the enunciated result.

Remark 1. (a) Applying Jensen's inequality to the convex function $\gamma(x)=$ $x^{2}$, we obtain ${ }^{s t} v^{2} \leq v^{2}$. Equality holds if and only if $\mathbb{L}=1$ or

$$
\frac{N l}{N} \sum_{i=1}^{M} \sum_{j=1}^{K} p_{i j l} \frac{\partial g_{r}^{s}(P)}{\partial p_{i j l}}
$$


does not depend on $l(l=1, \ldots, L)$.

(b) As the random variables

$$
2 n^{s t} D_{r}^{s}\left(\hat{P}_{X Y} \| P_{X} * P_{Y}\right) \text { and } n \sum_{i=1}^{M} \sum_{j=1}^{K} \frac{1}{p_{i . . . p_{. j}}}\left(\hat{p}_{i j .}-p_{i . . p_{. j .}}\right)^{2}
$$

converge in law to the same distribution under the hypothesis of independence and.

$$
Y^{\prime}=n^{\frac{1}{2}}\left[\left(\hat{p}_{11 .}-p_{1 . . p .1 .}\right), \ldots,\left(\hat{p}_{M K .}-p_{M . . p . K .}\right)\right] \underset{n_{\uparrow} \infty}{L} N\left[0, \sum_{l=1}^{L} \frac{N_{l}}{N} \Sigma(l)\right]
$$

If $P_{X Y}=\mathbb{P}_{X} * P_{Y}$ and $P_{X}=P_{1}, P_{Y}=P_{2}$ with $P_{1}$ and $P_{2}$ known, then

$$
T_{3}=n Y^{\prime} C Y=2 n^{s t} D_{r}^{s}\left(\hat{P}_{X Y} \| P_{X} * P_{Y}\right) \underset{n_{\uparrow} \infty}{L} \sum_{h=1}^{M K} \beta_{h} \chi_{1}^{2}
$$

where $\beta_{i}$ 's are the eigenvalues of the matrix $C \Sigma^{*}$, being

$$
C=\operatorname{diag}_{M K \times M K}\left[\frac{1}{p_{i . . p . j .}}\right]_{\substack{i=1, \ldots, M \\ j=1, \ldots, K}}, \Sigma^{*}=\sum_{l=1}^{L} \frac{N_{K}}{N} \Sigma(l)
$$

and the $\chi_{1}^{2}$ 's are independent (see Mardia et al, 1979, pp. 68).

\section{Tests of Independence}

Let $X$ and $Y$ be two random variables with joint distribution function $F(x, y)$, and let $F_{X}$ and $F_{Y}$ be the marginal distribution function of $X$ and $Y$, respectively. In this section we study some tests of the hypotheses of independence, namely, $H_{0}: F(x, y)=F_{X}(x) F_{Y}(y)$ for all $(x, y) \in \mathbb{R}^{2}$, against the alternative $H_{1}: F(x, y) \neq F_{X}(x) F_{Y}(y)$ for some $(x, y)$.

We suppose that we have $n$ observations on $(X, Y)$. Let us divide the space of values assumed by $X$ (the real line) into $M$ mutually intervals $I_{1}, \ldots, I_{M}$. Similarly, the space of values of $Y$ is divided into $K$ disjoints intervals $J_{1}, \ldots, J_{K}$. Let $\hat{p}_{i j}$ be the observed frequency of cell $(i, j)$, and let $p_{i j}=P((X, Y) \in$ 
$\left.I_{i} \times J_{j}\right)=\mathbb{P}\left(X \in \mathbb{I}_{i}\right.$ and $\left.Y \in J_{j}\right)$, where $i=1, \ldots, M$ and $j=1, \ldots, \mathbb{K}$. Then the random vector $\left(n \hat{p}_{11}, \ldots, n \hat{p}_{M K}\right)$ has a multinomial distribution with parameters $\left(n ; p_{11}, \ldots, p_{M K}\right)$. The hypothesis to be tested is $\mathbb{H}_{0}: p_{i j}=p_{i . p . j}$ $i=1,2, \ldots, M, j=1, \ldots, K$, where $p_{i .}=\mathbb{P}\left(X \in \mathbb{I}_{i}\right)$ and $p_{. j}=\mathbb{P}\left(Y \in J_{j}\right)$.

We consider the statistics given in theorem 2

$$
T_{1}=\frac{2 n D_{r}^{s}\left(\hat{P}_{X Y} \| \hat{P}_{X} * \hat{P}_{Y}\right)}{r}
$$

If $H_{0}$ is true, then $T_{1}$ will be small. Thus a large value of $T_{1}$ indicates data less compatible with the null hypothesis. Hence for large $n$ a level $\alpha$ test is given y

$$
\Phi\left(\hat{p}_{11}, \ldots, \hat{p}_{M K}\right)= \begin{cases}1 & \text { if } T_{1}>\chi_{(M-1)(K-1), \alpha}^{2} \\ 0 & \text { otherwise }\end{cases}
$$

The theorem 1 can be used to evaluate the asymptotic power of the previous test when $\left(p_{11}, \ldots, p_{M K}\right)$ is not equal to $\left(p_{1 . p .1}, \ldots, p_{M . p . k}\right)$.

A second possibility appears when the hypothetical probabilities $p_{i}$. and $p_{. j}$ specifying the marginal distributions may be known, in which case we are required to examine whether the probabilities $p_{i j}\left[=P\left(X=x_{i}, Y=y_{j}\right)\right]$ could be constructed by the law

$$
p_{i j}=p_{i .} p_{. j}
$$

Let $X$ and $Y$ be two random variables with joint distribution function $F(x, y)$, and let $F_{X}$ and $F_{Y}$ be the known marginal distribution of $X$ and $Y$ respectively. A test of hypothesis to test $H_{0}: F(x, y)=F_{X}(x) F_{Y}(y)$ for all $(x, y) \in \mathbb{R}^{2}$ against the alternative $\mathbb{H}_{1}: F(x, y) \neq F_{X}(x) F_{Y}(y)$ for some $(x, y) \in \mathbb{R}^{2}$ can be constructed

If we have $n$ observations on $(X, Y)$ obtained by random sampling, we consider the statistics

$$
T_{2}=\frac{2 n D_{r}^{s}\left(\hat{P}_{X Y} \| P_{X} * P_{Y}\right)}{r}
$$

If $H_{0}$ is true, then $T_{2}$ is asymptotically chi-square distributed with $M K-1$ degrees of freedom. Hence for large $n$, one must reject $H_{0}$ at a level $\alpha$ if $T_{2}>$ $\chi_{M K-1, \alpha}^{2}$ 
On the other hand, if we have $n$ observations on $(X, Y)$ obtained by stratified random sampling with proportional allocation, we consider the statistics $T_{3}$ given in remark $1(b)$. If $H_{0}$ is true, then $T_{3}$ will be small. Thus a large value of $T$ indicates data less compatible with the null hypothesis. Hence for large $n$, when $T_{3}=t$, one must reject $h_{0}$ at level $\alpha$ if

$$
P\left[\sum_{h=1}^{M K} \beta_{h} \chi_{1}^{2}>t\right] \leq \alpha
$$

where $\beta_{h}$ 's are given in remark $1(b)$. This probability can be computed using the methods given by Kotz et al (1967).

\section{References}

[1] P. J. Bickel, and K. A. Doksum, Mathematical Statistics Holden-Day, Inc. Oakland, California, (1977).

[2] M. A. Gil, "A note on stratification and gain in precision in estimating diversity from large samples", Commur. Statist. ( Theory and Methods) 18(4), (1989), 1521-1526.

[3] S. Kotz, N. M. Johnson, and D. W. Boid, "Series representation of distributions of quadratic forms in normal variables", I. Central Case, AMS 823-837, (1967).

[4] S. Kullback, and A. Leibler, "On the Information and Sufficiency", Ann. Math. Statist., 27, (1951), 986-1005.

[5] K. V. Mardia, J. T. Kent, and J. M. Bibby, "Multivariate analysis", Academic Press, (1979).

[6] M. L. Menendez, D. Morales, L. Pardo, and M. Salicru, "Some Applications of $(r, s)$ Directed divergences", To appear in Utilitos Mathcmatica.

[7] D. Morales, L. Pardo, "Statistical Application of Hypoentropy Measure to Populations Diversity Analisis", To appear in Trabajos de Estadística, (1991).

[8] T. K. Nayak, "Applications of entropy functions in measurement and analysis of diversity", Ph. D. thesis. Un. Pittsburgh. Ed. Un. Microfilm International, (1983).

[9] T. K. Nayak, "On Diversity Measures based on Entropy Functions", Commun. Statis.Theory and Methods, 14, 1, (1985), 203-215.

[10] L. Pardo, and D. Morales, "An Index of Diversity in Stratified Random Sampling based on the Hypoentropy Measure", Qüestiio, 14, 1,2,3,(1991), 11-25

[11] L. Pardo, J. A. Pardo, M. L. Menendez, "Unified $(r, s)$-Entropy as an index of Diversity", To appear in. Journol of the FranKlin Institute

[12] C. R. Rao, "Linear Statistcal Inference and its Applications", John Wiley, New York, (1973).

[13] A. Renyi, "On Measures of Entropy and Information". Procd. 4th Berkeley Symp. Math. Statist. and Prob., 1, (1961), 547-561.

[14] M. Salicrú, "Asy mptotic properies of $(r, s)$-Directed Divergence in a Stratified Sampling", (Communicated), (1991). 
[15] R. J. Serfling, "Approximation theorems of Mathematical statistics", Wiley, New York, (1980).

[16] C. Shannon, "A mathematical Theory of Comunications", Bell. System. Tech. J. 27, (1948), 379-423.

[17] B. D. Sharma and D. P. Mittal, "New Nonadditive Measures of Entropy for Discrete Probability Distributions", J. Math. Sci., 10, (1975), 28-40.

[18] K. Zografos, K. Ferentinos, and T. Papaioannou, " $\varphi$-Divergence Statistics: Sampling Properties and Multinomial Goodness of fit and Divergence Tests", Commun. Statist. (Theory Meth.), 19(5), (1990), 1785-1802.

Departamento de Estadistica e I.O., Facultad de Matemáticas, Universidad Complutense de Madrid, 28040 MADRID (SPAIN).

Departamento de Estadistica e I.O., Facultad de Matemáticas, Universidad Complutense de Madrid, 28040 MADRID (SPAIN).

Departamento de Estadística, Universidad de Barcelona, 08028 BARCELONA (SPAIN).

Departamento de Matemática aplicada, E. T. S. de Arquitectura, Universidad Politécnica de Madrid, 28040 MADRID (SPAIN). 